SLAC-PUB-10389

March 2004

\title{
Warped Higgsless Models with IR-Brane Kinetic Terms * ${ }^{*}$
}

\author{
H. Davoudiasl ${ }^{1, a}$, J.L. Hewett ${ }^{2, b}$, B. Lillie ${ }^{2, c}$, and T.G. Rizzo ${ }^{2, d}$ \\ ${ }^{1}$ School of Natural Sciences, Institute for Advanced Study, Princeton, NJ 08540 \\ ${ }^{2}$ Stanford Linear Accelerator Center, Menlo Park, CA, 94025
}

\begin{abstract}
We examine a warped Higgsless $S U(2)_{L} \times S U(2)_{R} \times U(1)_{B-L}$ model in 5 - $d$ with $\operatorname{IR}(\mathrm{TeV})$-brane kinetic terms. It is shown that adding a brane term for the $U(1)_{B-L}$ gauge field does not affect the scale $(\sim 2-3 \mathrm{TeV})$ where perturbative unitarity in $W_{L}^{+} W_{L}^{-} \rightarrow W_{L}^{+} W_{L}^{-}$is violated. This term could, however, enhance the agreement of the model with the precision electroweak data. In contrast, the inclusion of a kinetic term corresponding to the $S U(2)_{D}$ custodial symmetry of the theory delays the unitarity violation in $W_{L}^{ \pm}$scattering to energy scales of $\sim 6-7 \mathrm{TeV}$ for a significant fraction of the parameter space. This is about a factor of 4 improvement compared to the corresponding scale of unitarity violation in the Standard Model without a Higgs. We also show that null searches for extra gauge bosons at the Tevatron and for contact interactions at LEP II place non-trivial bounds on the size of the IR-brane terms.
\end{abstract}

\footnotetext{
*Work supported in part by the Department of Energy, Contract DE-AC03-76SF00515

†e-mails: $\quad{ }^{a}$ hooman@ias.edu, ${ }^{b}$ hewett@slac.stanford.edu, ${ }^{c}$ lillieb@slac.stanford.edu, and drizzo@slac.stanford.edu
} 


\section{Introduction}

As we enter the era of the LHC experiments, it is appropriate to examine the features of various approaches to Electroweak Symmetry Breaking (EWSB). One of the latest attempts for describing EWSB is the proposal of Refs. [1, 2]. In this approach, a judiciously chosen set of boundary conditions in a $5-d$ Higgsless $S U(2)_{L} \times S U(2)_{R} \times U(1)_{B-L}$ model gives rise to a pattern of gauge boson masses and couplings that are similar to those obtained in the Standard Model (SM) via a Higgs doublet condensate. The geometry of this model is based on the Randall-Sundrum (RS) hierarchy solution [3], where two branes reside at the boundaries of a 5-d Anti-de Sitter space ${ }^{\ddagger}$. In this scenario, the boundary conditions give rise to the breaking chain $S U(2)_{R} \times U(1)_{B-L} \rightarrow U(1)_{Y}$ at the Planck scale with the subsequent breaking $S U(2)_{L} \times U(1)_{Y} \rightarrow U(1)_{Q E D}$ at the $\mathrm{TeV}$ scale. After the Planck scale symmetry breaking occurs, a global $S U(2)_{L} \times S U(2)_{R}$ symmetry remains in the brane picture; this breaks on the $\mathrm{TeV}$-brane to a diagonal group $S U(2)_{D}$ corresponding to the custodial $S U(2)$ symmetry present in the SM [8].

It has been shown [2, 5, 8, that due to the presence of the $S U(2)_{D}$ custodial symmetry, this Warped Higgsless Model (WHM) enjoys good agreement with precision EW data at the level of a few percent. However, it has been argued [6, 7] and demonstrated [9] that the region of parameter space in the WHM that results in good agreement with the EW data leads to perturbative unitarity violation (PUV) in $W_{L}^{+} W_{L}^{-}$scattering at energies of order $\sim 2-3 \mathrm{TeV}$. Furthermore, a scan of the parameter space of the WHM shows that the scale of perturbative unitarity violation is never significantly raised, even in those regions where comparisons with the precision measurements are anticipated to be quite poor [9]. To restore unitarity in gauge boson scattering, additional new physics is required at or below the RS

\footnotetext{
${ }^{\ddagger}$ In the RS background, holographic arguments based on the AdS/CFT correspondence [4] have been useful in elucidating the features of the Higgsless theory [2, 5, 6, 7.
} 
cutoff of the effective theory on the TeV-brane. Even though this does not by itself rule out the model, it suggests that interactions in the gauge sector are problematic above the $\mathrm{TeV}$ scale.

To address some of these issues, the authors of Ref. [10] have examined the effects of including $\operatorname{IR}(\mathrm{TeV})$-brane terms for the $U(1)_{B-L}$ and the custodial $S U(2)_{D}$ gauge symmetries. It is well-known that the introduction of brane terms can alter the couplings and masses of the corresponding Kaluza-Klein (KK) tower states [11] and this would hence affect their contributions to the precision EW observables and to $W_{L}^{+} W_{L}^{-}$scattering. These authors concluded that the addition of the $U(1)_{B-L}$ brane term could lead to improved agreement with the EW data, and, in addition, lowers the mass of the lightest KK state to $\sim 300$ GeV. Light KK states are generically expected to help restore perturbative unitarity in high energy gauge boson scattering, however the analysis of Ref. [10] did not quantify this point.

In this paper, we also study the effects of the IR-brane kinetic terms associated with both the $U(1)_{B-L}$ and $S U(2)_{D}$ symmetries; here, we pay particular attention to low energy perturbative unitarity violation. For the $U(1)_{B-L}$ boundary term, we find that the scale of PUV in the model is independent of the size of the brane term. We also demonstrate that increasing the ratio of the $5-d$ couplings, $\kappa \equiv g_{5 R} / g_{5 L}$, improves the agreement with the tree-level SM relations in the electroweak sector, but lowers the scale at which perturbative unitarity is violated, similar to our previous results [9]. In the case of the $S U(2)_{D}$ kinetic term, we find that perturbative unitarity violation in $W_{L}^{+} W_{L}^{-}$scattering could be delayed to center of mass energies of order $\sim 6-7 \mathrm{TeV}$. However, agreement with the tree-level SM relations is rather poor, with the disparity worsening as the size of the $S U(2)_{D}$ brane term increases. In addition, we compare the predictions for the lowest lying gauge KK state to the searches for new gauge bosons at the Tevatron Run I and II and for contact interactions at LEP II and find that the collider bounds restrict the potential size of the IR-brane kinetic 
terms. However, these collider bounds allow for the PUV scale to approach $6-7 \mathrm{TeV}$.

We describe our setup in the next section. The EW and collider constraints are discussed in section 3. Perturbative unitarity in this model is the subject of section 4 and our concluding remarks are given in section 5 .

\section{The Model}

Here, we briefly discuss the modifications induced in our earlier analysis [9] due to the presence of the $U(1)_{B-L}$ and $S U(2)_{D}$ brane terms; these changes are quite straightforward. We employ the notation introduced in our previous work. In what follows, when we consider the effects of the $U(1)_{B-L}$ kinetic term we also include the UV-brane terms associated with the $S U(2)_{L}$ and $U(1)_{Y}$ symmetries in our analysis; these UV kinetic terms were included in our earlier results. However, for simplicity, we omit the UV terms in our study of the $S U(2)_{D}$ kinetic term.

The introduction of new kinetic terms on the $\mathrm{TeV}$ brane leads to a shift in the original action (given in Eq.(4) of Ref.[9]) by an amount

$$
\delta S_{\text {brane }}=\int d^{4} x d y \sqrt{-g} \delta\left(y-\pi r_{c}\right)\left[-\frac{1}{4} r_{c} c_{B} F_{B-L}^{2}-\frac{1}{4\left(g_{5 L}^{2}+g_{5 R}^{2}\right)} r_{c} c_{D}\left(g_{5 R} F_{L}+g_{5 L} F_{R}\right)^{2}\right]
$$

with $g_{5 L(R)}$ being the $5-d S U(2)_{L(R)}$ gauge coupling, $\pi r_{c}$ is the brane separation in the RS

model, and $c_{B, D}$ are dimensionless parameters which quantify the size of the IR-brane kinetic terms. Here $F_{B-L}$ is the field strength tensor for the $U(1)_{B-L}$ gauge field, and similarly $F_{L, R}$ corresponds to $S U(2)_{L, R}$. For later purposes it is convenient to introduce the quantities $\delta_{B, D} \equiv k r_{c} c_{B, D} / 2$ as in our earlier analysis where $k$ is the RS curvature parameter. We next observe that a non-zero value for $\delta_{B}$ will alter the $\partial_{z} B=0$ boundary condition [2] on the TeV brane; instead, we now find $\partial_{z} B-\delta_{B} x_{n}^{2} k \epsilon B=0$, where $x_{n}$ represents the roots defining 
the KK spectra, $\epsilon \equiv e^{-\pi k r_{c}}$, and $B$ represents the $U(1)_{B-L}$ gauge field. A similar shift is observed in the case of the combination of fields associated with the $S U(2)_{D}$ brane term, i.e., $\partial_{z}\left(g_{5 R} A_{L}+g_{5 L} A_{R}\right)-\delta_{D} x_{n}^{2} k \epsilon\left(g_{5 R} A_{L}+g_{5 L} A_{R}\right)=0$. Solving these new boundary conditions leads to alterations of the wavefunction coefficients as well as the eigenvalue equations for the KK tower masses. It is important to note, however, that the $W^{ \pm} \mathrm{KK}$ tower masses and couplings are left unaltered by a non-zero value of $\delta_{B}$, but are modified by $\delta_{D}$.

In calculating the couplings to both fermions and $W^{ \pm}$pairs for the photon, the $Z$, as well as the rest of the KK tower states, one of the dominant effects due to the new brane terms is the shift in the normalizations of the $W_{n}^{ \pm}$and $Z_{n}$ wavefunctions. These normalizations now pick up additional terms; for the case of the $Z_{n}$, in comparison to our earlier result (Eq.(50) of Ref. 9] ); we now obtain:

$$
\begin{aligned}
N_{Z_{n}} & =\int_{R}^{R^{\prime}} d z \frac{R}{z}\left\{\left|\chi_{L}^{n}(z)\right|^{2}\left(2+c_{L} r_{c} \delta(z-R)\right)+2\left|\chi_{R}^{n}(z)\right|^{2}+2\left|\chi_{B}^{n}(z)\right|^{2}\right. \\
& +c_{Y} r_{c} \frac{\left|\kappa \chi_{B}^{n}(z)+\lambda \chi_{R}^{n}(z)\right|^{2}}{\kappa^{2}+\lambda^{2}} \delta(z-R)+c_{B} r_{c}\left|\chi_{B}^{n}(z)\right|^{2} \delta\left(z-R^{\prime}\right) / \epsilon \\
& \left.+c_{D} r_{c} \frac{\left|\kappa \chi_{L}^{n}(z)+\chi_{R}^{n}(z)\right|^{2}}{1+\kappa^{2}} \delta\left(z-R^{\prime}\right) / \epsilon\right\},
\end{aligned}
$$

where $\chi_{i}^{n}$ are the wavefunctions for the relevant gauge KK state, and $\lambda$ is defined as the ratio $\lambda \equiv g_{5 B} / g_{5 L}$. A similar shift in the $W_{n}^{ \pm}$normalization also occurs,

$$
\begin{aligned}
N_{W_{n}} & =\int_{R}^{R^{\prime}} d z \frac{R}{z}\left\{\left|\chi_{L}^{n}(z)\right|^{2}\left(2+c_{L} r_{c} \delta(z-R)\right)+2\left|\chi_{R}^{n}(z)\right|^{2}\right. \\
& \left.+c_{D} r_{c} \frac{\left|\kappa \chi_{L}^{n}(z)+\chi_{R}^{n}(z)\right|^{2}}{1+\kappa^{2}} \delta\left(z-R^{\prime}\right) / \epsilon\right\} .
\end{aligned}
$$

These new TeV brane terms also lead to additional contributions to the normalization of the 
photon wave function,

$$
N_{\gamma}=2 \pi r_{c} \alpha_{L}^{2}\left(\frac{\kappa^{2}+\lambda^{2}+\kappa^{2} \lambda^{2}}{\kappa^{2} \lambda^{2}}\right)\left\{1+\frac{1}{\pi k r_{c}} \frac{\kappa^{2} \lambda^{2} \delta_{L}+\kappa^{2} \delta_{B}+\left(1+\kappa^{2}\right) \lambda^{2} \delta_{D}+\left(\kappa^{2}+\lambda^{2}\right) \delta_{Y}}{\kappa^{2}+\lambda^{2}+\kappa^{2} \lambda^{2}}\right\}
$$

where $\alpha_{L}$ is a numerical constant which is determined from the boundary conditions and appears in the KK decomposition of the $A_{L}^{3}$ gauge field. Due to the abelian nature of the $U(1)_{B-L}$ group, new brane term contributions to the $W$ 4-point or gauge 3-point functions do not occur. However, such contributions are induced in the case of the $S U(2)_{D}$ brane term.

\section{Precision Measurements and Collider Bounds}

Our analysis now proceeds by analogy with our earlier work 9$]$ : we hold $M_{W, Z}$ as well as the UV-brane kinetic terms $\delta_{L, Y}$ fixed and explore the parameter space spanned by the parameters $\kappa$ and $\delta_{B, D}$.

We previously introduced the three different quantities related to the weak mixing angle: $\sin ^{2} \theta_{o s}=1-M_{W}^{2} / M_{Z}^{2}$, $\sin ^{2} \theta_{e g}=e^{2} / g_{W_{1}}^{2}$ (where $g_{W_{1}}$ is the coupling of the particle we identify as the $W$ to the SM fermions), and $\sin ^{2} \theta_{\text {eff }}$, which is defined at the $Z$-pole. All three must take on the same value at the tree-level in the SM. They can differ significantly in the present scenario; however, there are preferred parameter space regions, i.e., when $\kappa$ is large [9], that yield consistent values. The first question to address here is the variation of $\sin ^{2} \theta_{\text {eg,eff }}$ with respect to the fixed on-shell value, $\sin ^{2} \theta_{o s}$, as $\delta_{B, D}$ are allowed to change for fixed $\kappa$. The results of this analysis are shown in Fig. 1, In the top panel we observe that $\sin ^{2} \theta_{\text {eff }}$ is $\delta_{B}$-independent, which we have verified analytically, while $\sin ^{2} \theta_{e g}$ increases as $\delta_{B}$ increases. In fact we see that for $\kappa=1(3)$ excellent agreement between the on-shell and 
effective values is obtained when $\delta_{B} \simeq 8(10)$. Overall, however, the case $\kappa=3$ yields more consistent values, as in our earlier work, due to the large separation between the quantities $\sin ^{2} \theta_{\text {os }}$ and $\sin ^{2} \theta_{\text {eff }}$ when $\kappa=1$. Clearly, a non-vanishing value of $\delta_{B}$ does help to bring the values of the various definitions of $\sin ^{2} \theta$ into agreement. In contrast, in the bottom panel we see that as $\delta_{D}$ increases both of the different $\sin ^{2} \theta$ values shrink in size and move away from the on-shell value thus getting further from the SM limit. Of course the $\kappa=3$ values remain closer to the SM than do those for $\kappa=1$, but in all cases the agreement is poor.

Since $\delta_{B, D}$ shift the the $\sin ^{2} \theta_{e g}$ curves in opposite directions, it is interesting to see what happens when these brane terms are simultaneously nonzero. This can be seen in Fig. 2 for $\kappa=1$. For the range of $\delta_{D}$ of interest we see that we can always find a value of $\delta_{B}$ for which $\sin ^{2} \theta_{o s} \simeq \sin ^{2} \theta_{e g}$. Unfortunately, since $\sin ^{2} \theta_{\text {eff }}$ is $\delta_{B}$ independent, including this brane term does not bring this quantity into accord with the others for $\kappa=1$; larger values of $\kappa$ may help in this regard.

Another quantity of interest is the value of the overall strength of the $Z$ boson coupling, denoted as $\rho_{e f f}^{Z}$, and in particular, its deviation from unity, i.e., $\delta \rho_{\text {eff }}^{Z} \equiv \rho_{\text {eff }}^{Z}-1$. This deviation is related to the pseudo-oblique parameter $T^{*}$ as $T^{*} / \alpha \equiv \delta \rho_{\text {eff }}^{Z}$. The pseudooblique parameters are defined in such a way so that they all take on the value zero in the tree level SM. They are introduced to guide our thinking about the direction in parameter space which approaches the SM. We note that it is important not to confuse these pseudooblique parameters with the conventionally defined purely oblique $S, T, U$. The dependency of $\delta \rho_{\text {eff }}^{Z}$ on $\delta_{B, D}$ for two different values of $\kappa$ is shown in Fig. 3. Note that this parameter remains relatively small in magnitude for both values of $\kappa$ as long as either $\delta_{B, D}$ does not become too large.

The other pseudo-oblique parameters $S^{*}, U^{*}$, as defined in Ref. 9], are also functions 

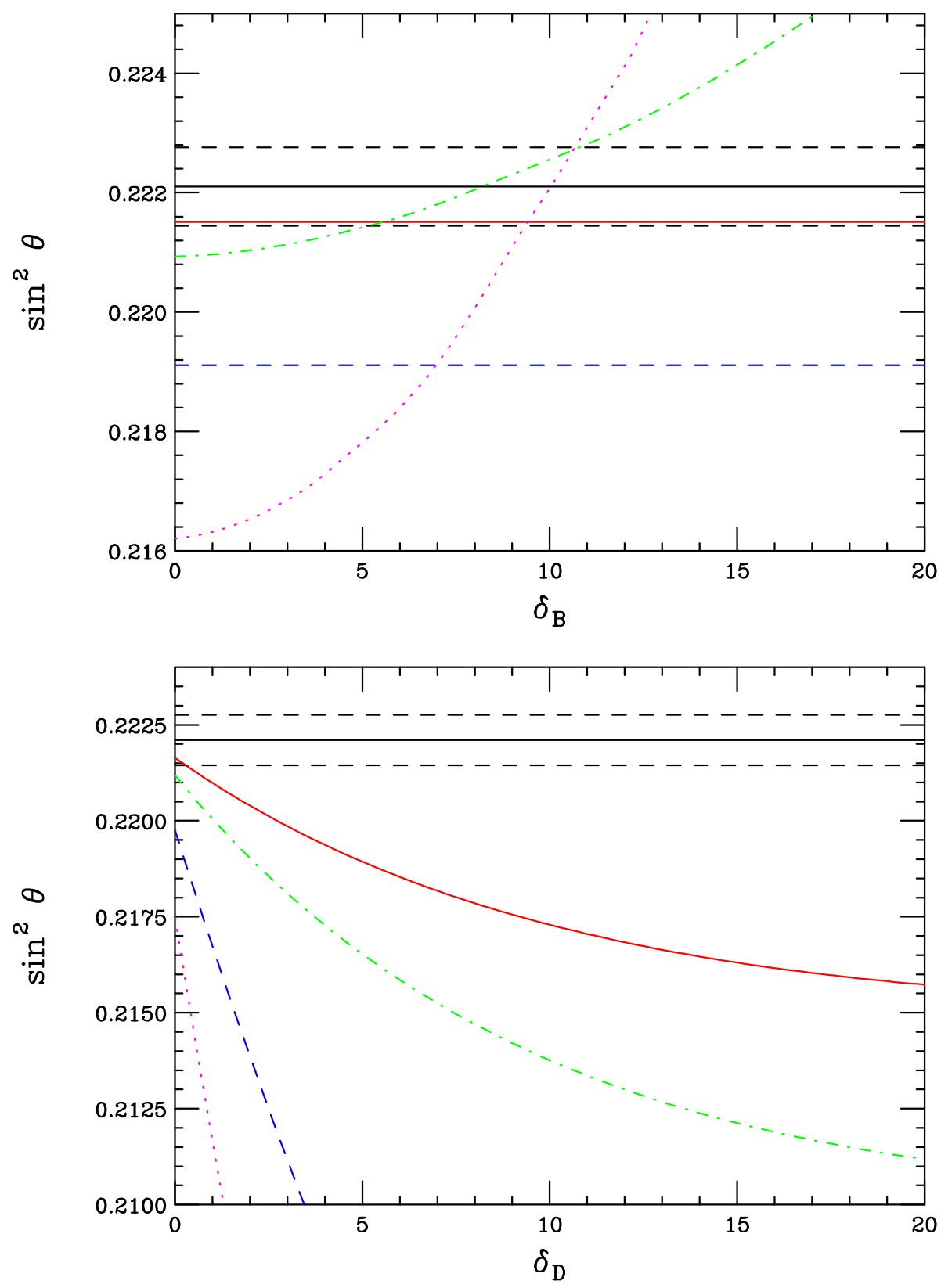

Figure 1: $\sin ^{2} \theta$ in each of the three definitions as a function of $\delta_{B, D}$. The black horizontal solid and dashed curves correspond to the on-shell value $\pm 1 \sigma$, the solid red (dashed blue) curve represents $\sin ^{2} \theta_{\text {eff }}$ for $\kappa=3(1)$ while the dash-dotted green (dotted magenta) curve is for $\sin ^{2} \theta_{e g}$. The top (bottom) panel illustrates the effects of including the $U(1)_{B-L}\left(S U(2)_{D}\right)$ kinetic term. We take only one IR kinetic term to be non-vanishing at a time. 


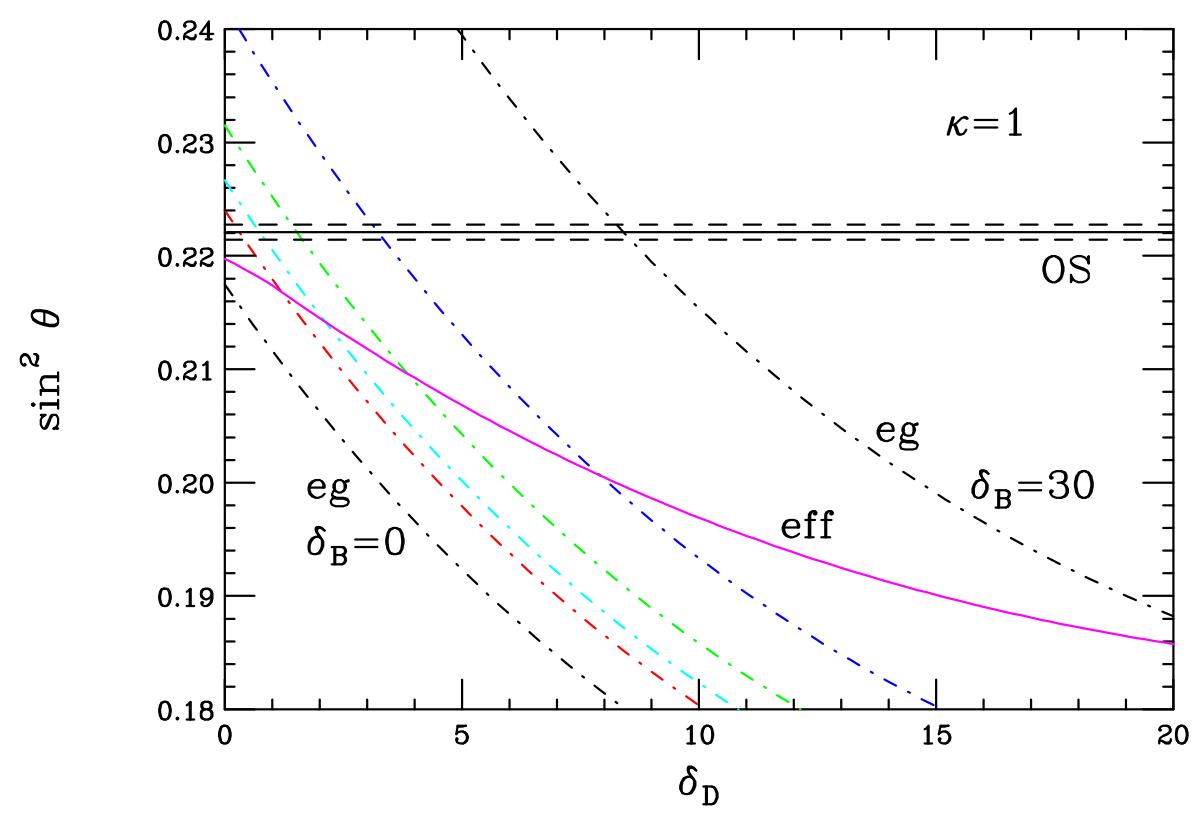

Figure 2: Same as in the previous figure but now with both $\delta_{B, D}$ nonzero for the case $\kappa=1$. The solid magenta curve is the value of $\sin ^{2} \theta_{\text {eff }}$ while the dash-dotted curves are all for $\sin ^{2} \theta_{e g}$ for, from left to right, $\delta_{B}=0,10,12,15,20$ and 30 , respectively.

of $\delta_{B, D}$ as shown in Fig. 固 For $U^{*}$, some values of $\delta_{B}$ improve the agreement with the SM limit, while $S^{*}$ tends away from its SM value. We see that, overall, smaller values of $\delta_{B}$ are again preferred. In the case of $\delta_{D}$ we see that both $S^{*}$ and $U^{*}$ move away from the SM limit with the shifts being much more significant in the case of $\kappa=1$.

Our approach to calculating the pseudo-oblique observables, $S^{*} T^{*} U^{*}$, differs from that of $S T U$ as calculated by Csaki et al.[10]. In our approach, we numerically fix the masses of the first charged and neutral gauge KK excitations to be those of the physical $W$ and $Z$ bosons observed at colliders. We use these as input to our analysis, together with the strength of the charged current coupling determined by $G_{F}$. From these the couplings and masses of all the gauge KK states can be obtained. The pseudo-oblique parameters are then defined in terms of observables via the $W$ mass, the invisible width of the $Z$ and the fermionic couplings determined at the $Z$-pole. $S^{*} T^{*} U^{*}$ are chosen to vanish at the tree-level in the SM. Csaki et al. choose a different scheme wherein the SM gauge couplings $g$ and 
$g^{\prime}$ are used as input parameters together with the usual relationship $1 / e^{2}=1 / g^{2}+1 / g^{\prime 2}$. This fixes $\sin ^{2} \theta$ and hence the couplings of the $W$ and $Z$. From this the $W$ and $Z$ and other KK masses, as well as their couplings, can be determined. The $S T U$ parameters in Ref. [10] can then be calculated as shifts in the masses as well as the wavefunctions and normalizations for the $W$ and $Z$. It is clear that these two sets of electroweak parameters probe different relationships between the masses and couplings of the $W$ and $Z$ described by distinct choices of input parameters. In either case they allow for a measure of how far the model predictions are from the tree level SM. However, without employing the full loop corrections and overcoming the problem of 'subtracting out' the Higgs loop effects (described in [9]) neither set of parameters can be directly compared with data.

To go further in the analysis of this model, we need to consider how non-zero values of $\delta_{B, D}$ lead to modifications of the KK spectra. Clearly, the $U(1)_{B-L}$ brane term does not influence the $W \mathrm{KK}$ tower so we turn our attention to the neutral KK states. The major effect of a non-zero $\delta_{B}$ on neutral KK states can be clearly seen in the upper panel of Fig. 5 for the case of $\kappa=1$; the same qualitative behavior occurs for other values of $\kappa$. Here we immediately observe that the single, non-degenerate states are unaffected while one member of the nearly degenerate paired states, the one which couples mainly to $B-L$, gets its mass reduced as $\delta_{B}$ is increased. The remaining member of the pair stays unaffected. In particular, we see that the state $Z_{2}$ becomes light (note that here, $Z_{1}$ is the lightest state and corresponds to the SM $Z$ ). Further increasing $\delta_{B}$ leads to the appearance of new sets of almost degenerate pairs of states. Including $\delta_{D}$ has the opposite effect in that the member of the pair which couples mainly to $T_{3 L}$ gets its mass lowered. The other states are only slightly affected. In the case of $S U(2)_{D}$, the charged KK states have all of their masses 

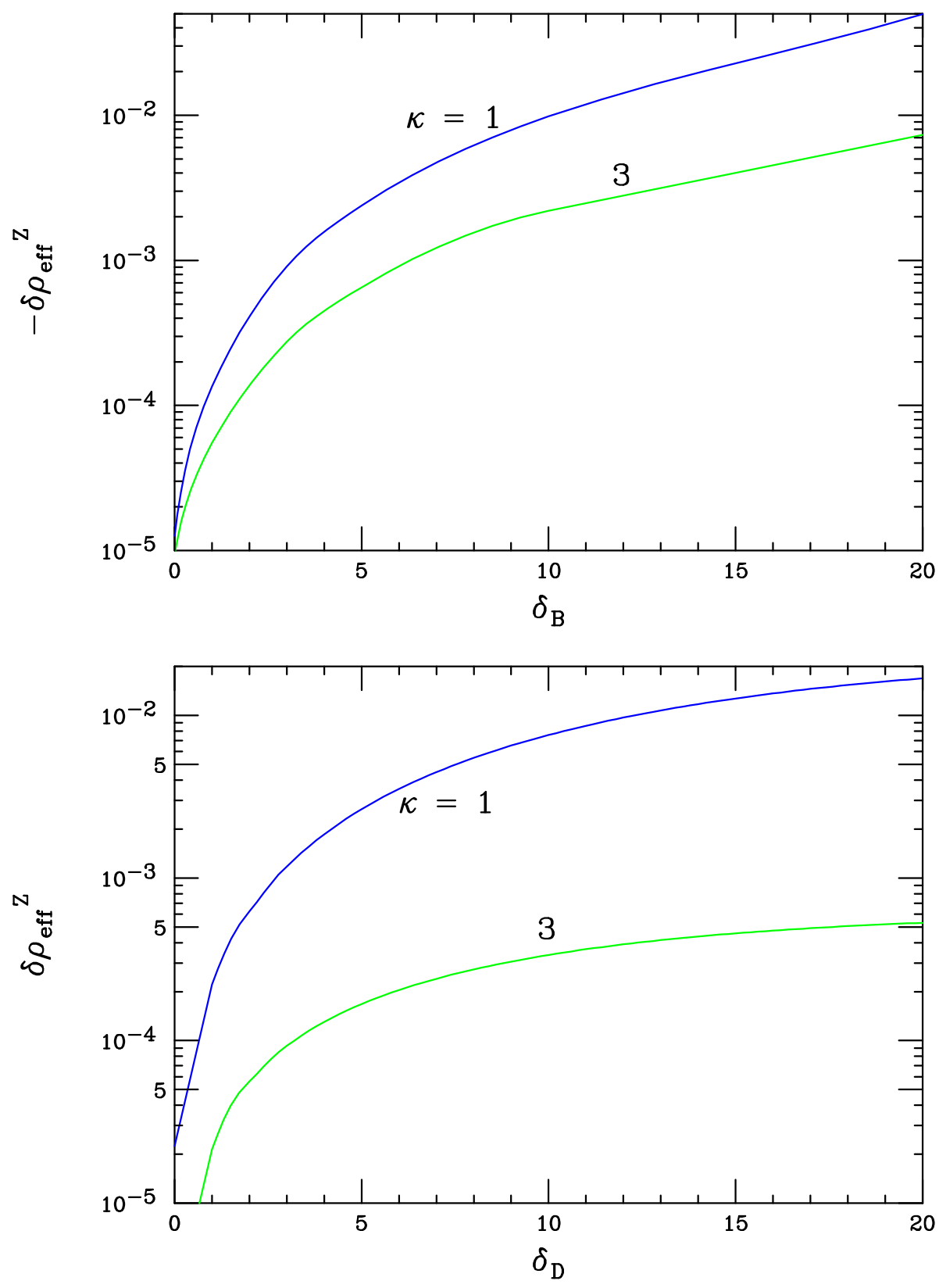

Figure 3: $\delta \rho_{\text {eff }}^{Z}$ as a function of $\delta_{B, D}$ for $\kappa=1$ and 3. We take only one IR kinetic term to be non-vanishing at a time. 

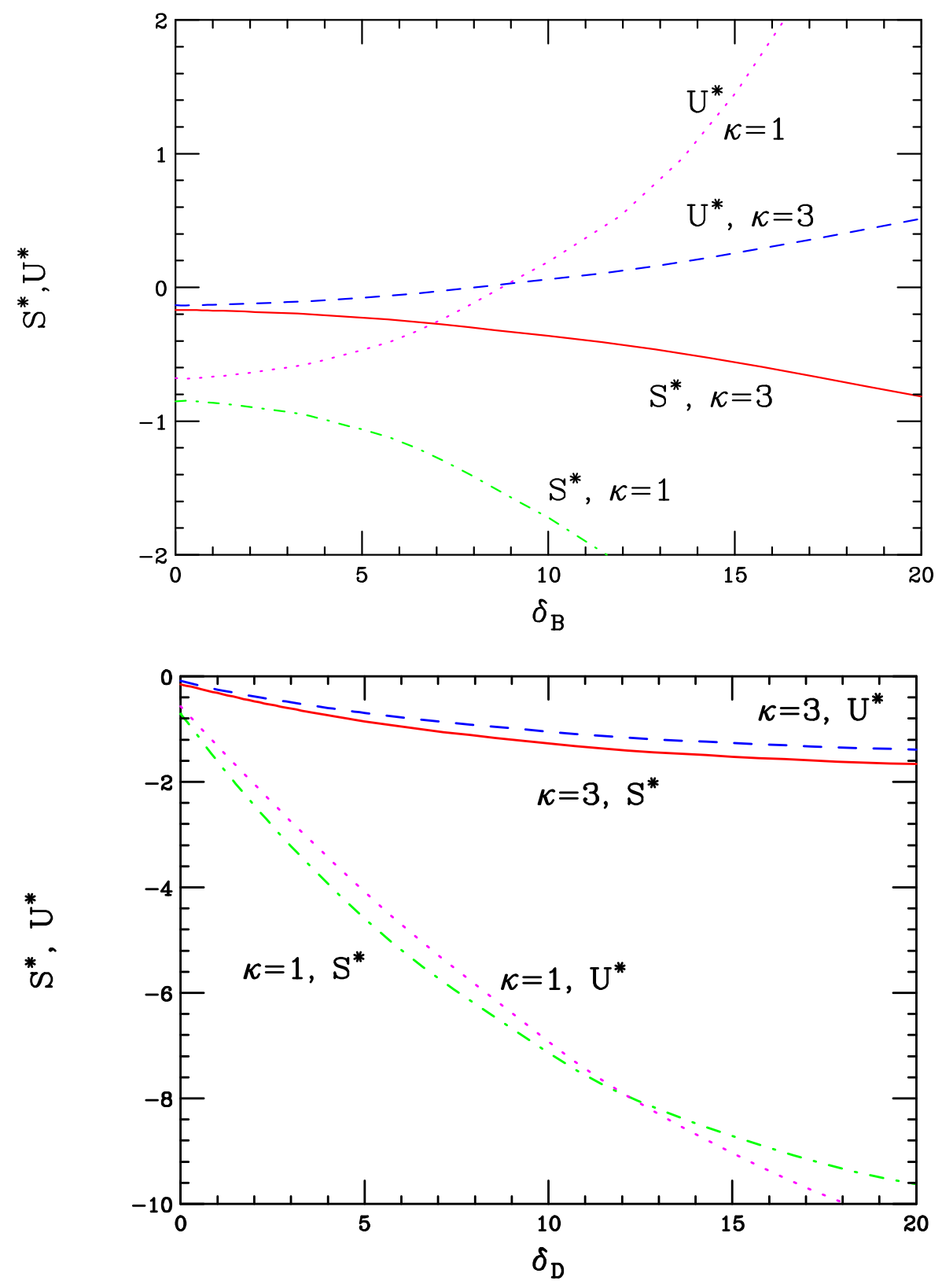

Figure 4: Values of the pseudo-oblique parameters $S^{*}$ (solid red, dash dotted green) and $U^{*}$ (blue dashed, dotted magenta) for of $\kappa=(3,1)$ as labeled as functions of $\delta_{B_{D}}$. We take only one IR kinetic term to be non-vanishing at a time. 
lowered in analogy with the falling curves in the lower panel ${ }^{\S}$. This figure demonstrates that the $U(1)_{B-L}$ and $S U(2)_{D}$ brane terms are at least partly doing what we had expected, i.e., lowering the KK masses so that the now lighter states can have a potentially greater influence on unitarity in $W_{L}^{+} W_{L}^{-}$scattering. They do, however, lower the masses of different sets of KK states and this is critical for unitarity considerations as we will see below.

One may wonder, since some of the KK states are becoming so light, if there are conflicts with direct searches for new vector bosons at the Tevatron as well as with indirect searches such as those for contact interactions at, e.g., LEP II. We recall that while the Tevatron experiments search for new resonances decaying into leptons via Drell-Yan production, the LEP bounds result from searches for deviations in cross sections and angular distributions from SM expectations below production threshold. In the case of the charged KK states, whose masses are lowered by the $S U(2)_{D}$ brane terms, the best limit comes from the Run I search at the Tevatron [12. The strongest bounds on the direct production of $Z^{\prime}$-like states come from Run II data using $200 \mathrm{pb}^{-1}$ of integrated luminosity [13, while indirect bounds on such states have been supplied by the LEPEWWG [14]. All of these sets of data have been employed in obtaining the results which follow. Figures 6 and 7 show the $\delta_{B, D}$ dependence of the lightest $\mathrm{KK}$ excitation mass for $\kappa=1,3$ as well as the corresponding bounds on this state from LEP II and the Tevatron. The non-trivial nature of these bounds arises from the modification in the $W_{2}$ and $Z_{2}$ couplings as $\delta_{B, D}$ are varied. Note that in the case of an $S U(2)_{D}\left[U(1)_{B-L}\right]$ brane term, the best limit from the Tevatron arises from constraints on $W^{\prime}\left[Z^{\prime}\right]$ production. The reason for this is that, in the case of $S U(2)_{D}$, both $W$ and $Z$ KK excitations may be light and the Tevatron constraints on $W^{\prime}$ production are generally stronger than those for $Z^{\prime}$ production since the cross section times leptonic branching fraction is larger in the $W^{\prime}$ case. For both values of $\kappa$ we again see that smaller values of $\delta_{B, D}$

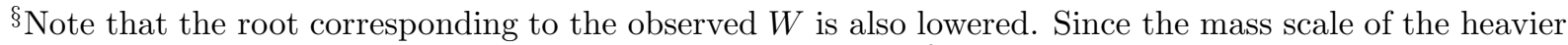
KK states is obtained by matching this root to $M_{W}$, the mass of the neutral states rises.
} 

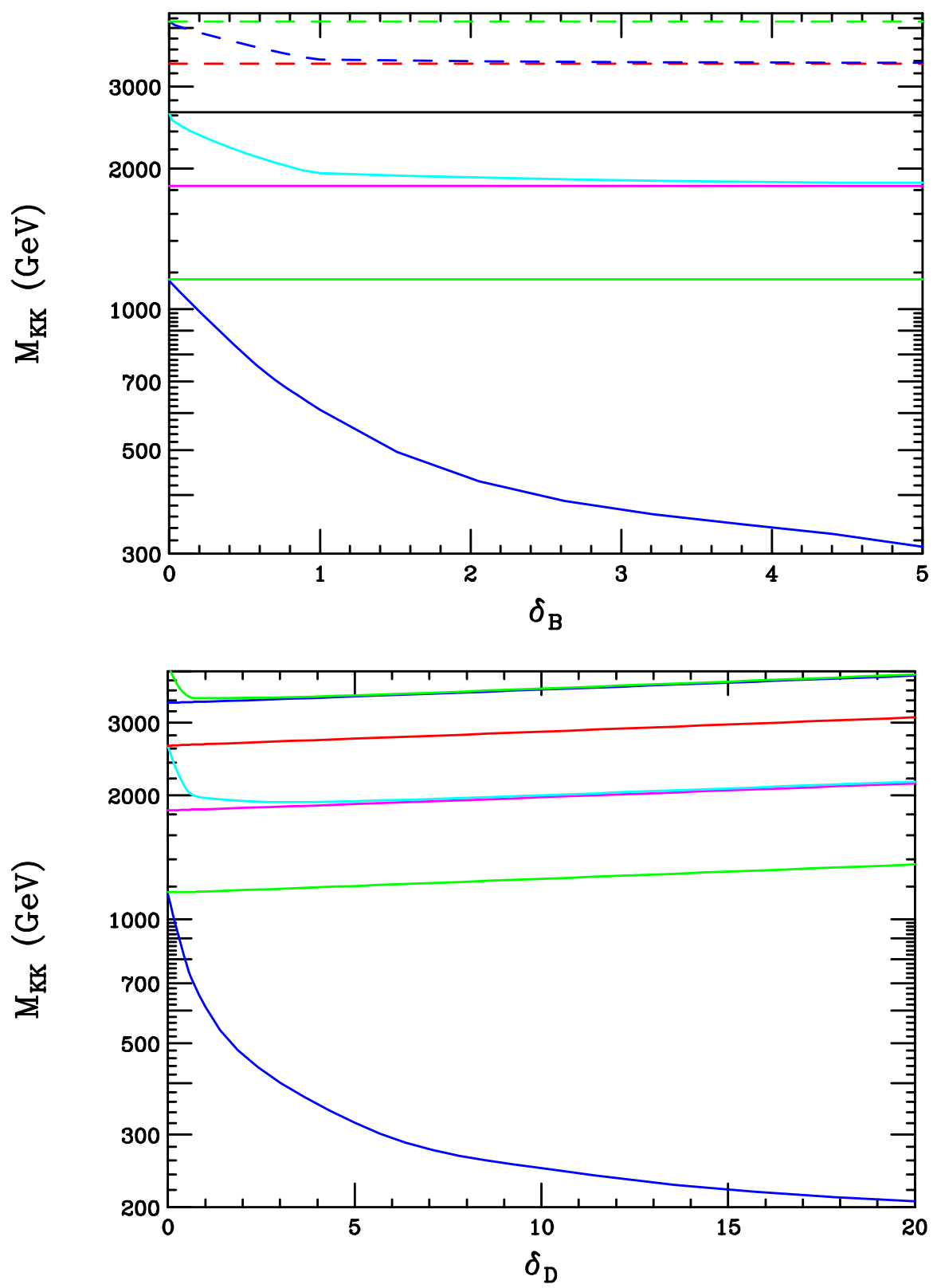

Figure 5: Behavior of the neutral KK mass spectrum as a function of $\delta_{B, D}$. From bottom to top on the left the curves correspond to the states $Z_{2,3, \ldots} . \kappa=1$ has been assumed. We take only one IR kinetic term to be non-vanishing at a time. 
are in better agreement with the data. Note that while the Tevatron bounds are somewhat sensitive to the assumption that all the SM fermions are localized close to the Planck brane due to possible variations in the width of the $W_{2}$ and $Z_{2}$, this is not true for those from LEP. For example, one can imagine that for model building purposes, the right-handed top-quark might be moved away from the Planck brane; this could significantly alter the bounds from the Tevatron but those from LEP II would remain intact.

As we will see below, the masses of the first $W$ and $Z$ KK excitations must be relatively light, $\leq 1 \mathrm{TeV}$, for there to be any impact on PUV. Though their couplings to the SM fermions are somewhat reduced, such states will not escape detection at the LHC and may even be observed in the near future at the Tevatron. The first neutral KK state may be sufficiently light to be produced on resonance at a $\mathrm{TeV}$-scale linear collider.

\section{$4 \quad$ Unitarity in $W_{L}^{+} W_{L}^{-}$Scattering}

We will now investigate the question of whether perturbative unitarity is preserved in this model. As before, we examine the amplitude for the $W_{L}^{+} W_{L}^{-} \rightarrow W_{L}^{+} W_{L}^{-}$elastic scattering process. In Ref. [1], two sum rules were derived that insure the cancellation of terms growing with energy at high energy. Here, we find that, as in our previous analysis, these sum rules are satisfied to good precision once sufficient KK states are included. However, these sum rules are technically only valid at infinite center of mass energy. If the scattering occurs at a finite value of $\sqrt{s}$, then the amplitude cannot receive contributions from states much heavier than $\sqrt{s}$. Therefore, we investigate the full amplitude in detail in the intermediate energy region, between $m_{Z}$ and the high-energy regime where the sum rules are valid. If unitarity is violated it will be in this region. Since the relevant expansion parameters, $M_{K K}^{2} / s$, are 

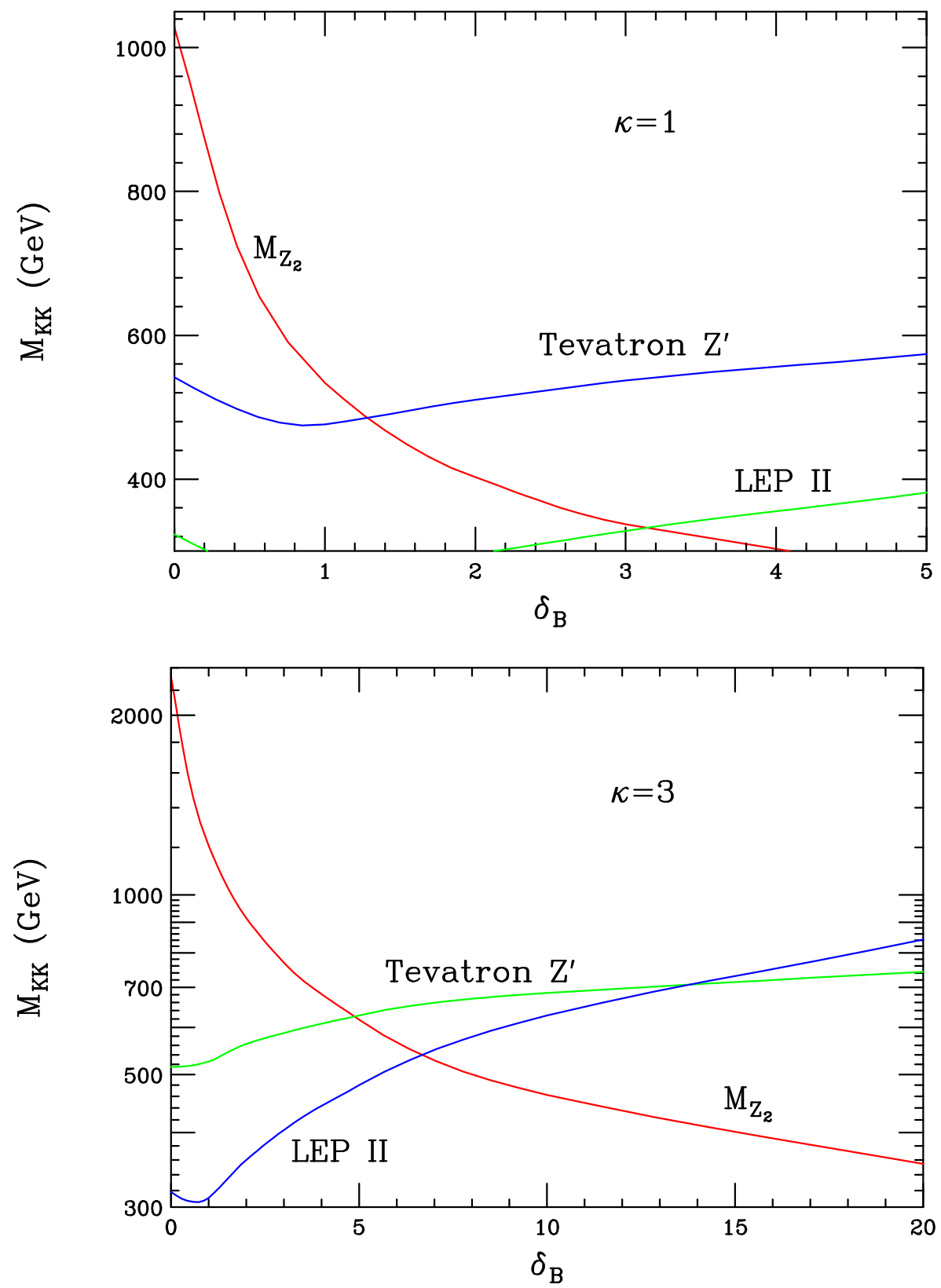

Figure 6: The predicted mass of the lightest KK excitation, the lower bound on the mass from the Run II Tevatron $Z^{\prime}$ searches as well as the lower bound from LEPII as a function of $\delta_{B}$, assuming $\delta_{D}=0$. The collider limits are discussed in detail in the text. 

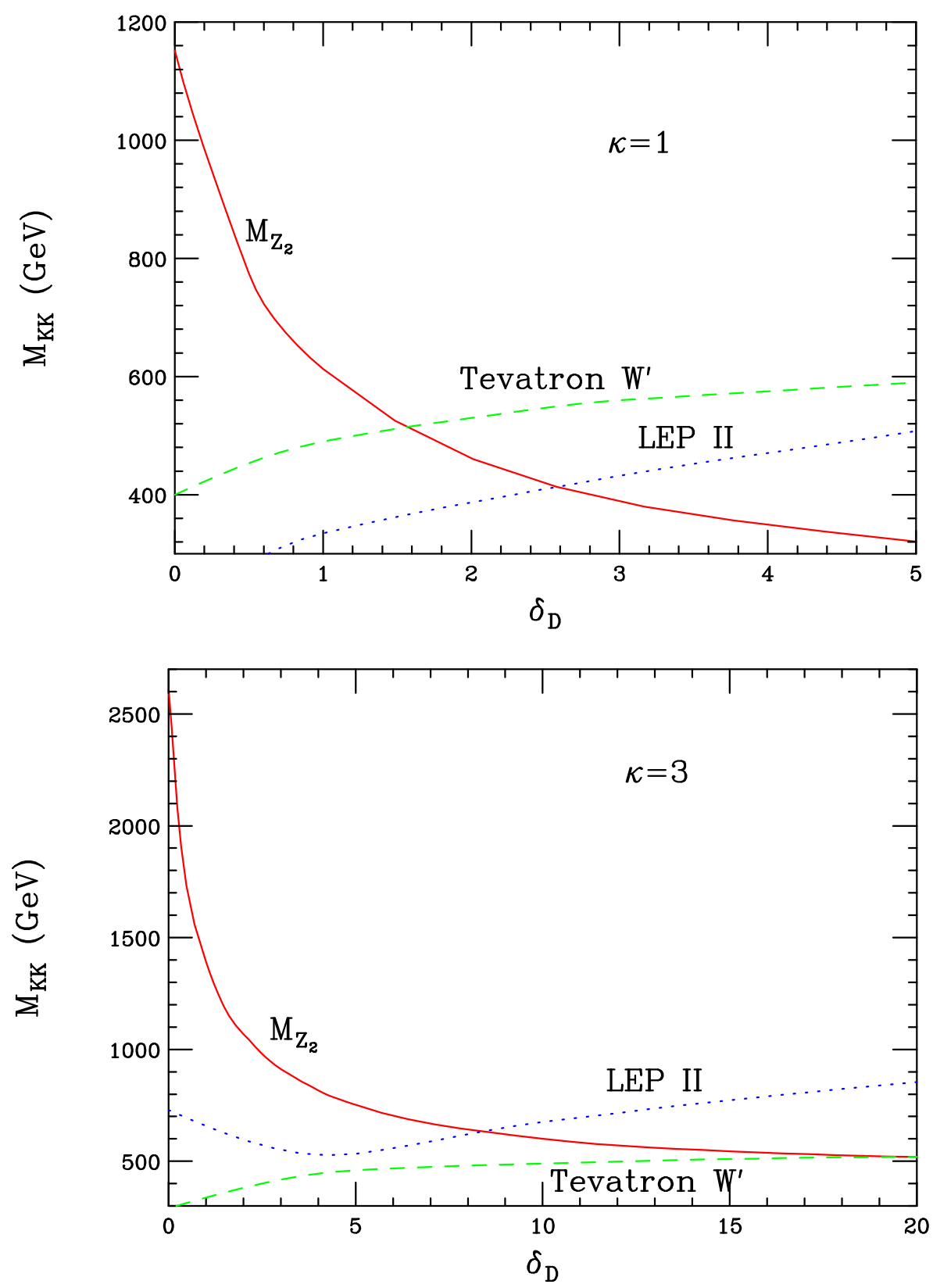

Figure 7: The predicted mass of the lightest KK excitation, the lower bound on the mass from the Run I Tevatron $W^{\prime}$ searches as well as the lower bound from LEPII as a function of $\delta_{D}$, assuming $\delta_{B}=0$. The collider limits are discussed in detail in the text. 
not small, we use the full tree-level amplitude from Ref. [15]. We numerically calculate the couplings using the $\delta_{B, D}$ generalized versions of Eq. (67) from Ref. 9]. We then numerically evaluate the amplitude and apply the partial wave unitarity condition $\left|\mathcal{R} e a_{0}\right| \leq 1 / 2$, where $a_{0}$ is the zeroth partial wave amplitude. The couplings were obtained independently on two different computing platforms, Maple and Mathematica. The partial wave amplitude was computed independently by three calculations, using Mathematica and Fortran. We have included all KK states with masses up to $10 \mathrm{TeV}$ and checked that the results are stable against including more states. For $\delta_{B} \neq 0, \delta_{D}=0$ we find that perturbative unitarity is violated and, furthermore, the scale of PUV is independent of $\delta_{B}$. For $\kappa=1$ the violation occurs at $3.8 \mathrm{TeV}$; for $\kappa=3$ it occurs at $1.9 \mathrm{TeV}$, close to the $\mathrm{SM}$ value. We have also checked the case $\delta_{L}=0, \delta_{B}=4, \kappa=1$ which roughly corresponds to the case studied in [10]; we found PUV at $3.15 \mathrm{TeV}$. For non-zero $\delta_{D}$, with all other $\delta_{i}$ set to zero, we find that the scale of PUV is increased over some of the parameter space, reaching energies $\sim 7 \mathrm{TeV}$, as displayed in Fig. 8 .

These results can be understood heuristically. Naively, one expects that the unitarity violations will be softened as the masses of the KK states contributing to unitarity restoration are lowered. Hence, one expects that a high value of $\delta_{B}$ will at least raise the scale of unitarity violation. However, note that gauge boson scattering is a fundamentally nonAbelian process. In the present model, it is therefore an $S U(2)_{L}$ process, and should not depend on the $U(1)_{B-L}$ dynamics. When $\delta_{B}$ is turned on, the mass of one state in a pair responds dramatically, while the other is unaffected. It is clear that the state that responds should be predominantly a hypercharge boson, with very little mixture of $W^{3}$ in its wavefunction. Indeed, we can write 9 the couplings of the neutral KK states to SM fermions as $\left(g_{Z_{n}} / c_{w}\right)\left(T_{3 L}^{f}-s_{n}^{2} Q^{f}\right)$. Calculation of the $s_{n}^{2}$ parameters confirms that the light state couples as a hypercharge boson. Numerically, we can look at the coupling of the light 
state to two $W_{L}$ bosons. At $\delta_{B}=0$ this coupling is a factor of 6 smaller than that for the next neutral KK state, which is predominantly $W^{3}$. As $\delta_{B}$ is increased to 20 the couplings of the two states become comparable. However, the light state still makes a negligible contribution to the part of the amplutude responsible for PUV. To see this, note that the PUV can be traced to incomplete cancellations in the term that grows linearly with s at high energies. The contribution of the $k$ th state to this sum rule is proportional to $m_{k}^{2} g_{11 k}^{2}$, so the light state has little effect. In the case where $\delta_{D}$ is non-vanishing, it is the other member of the degenerate KK pair whose mass is lowered. In this case, the light state then couples mostly to isospin, and is capable of significantly modifying the $W_{L}^{+} W_{L}^{-}$scattering amplitude.

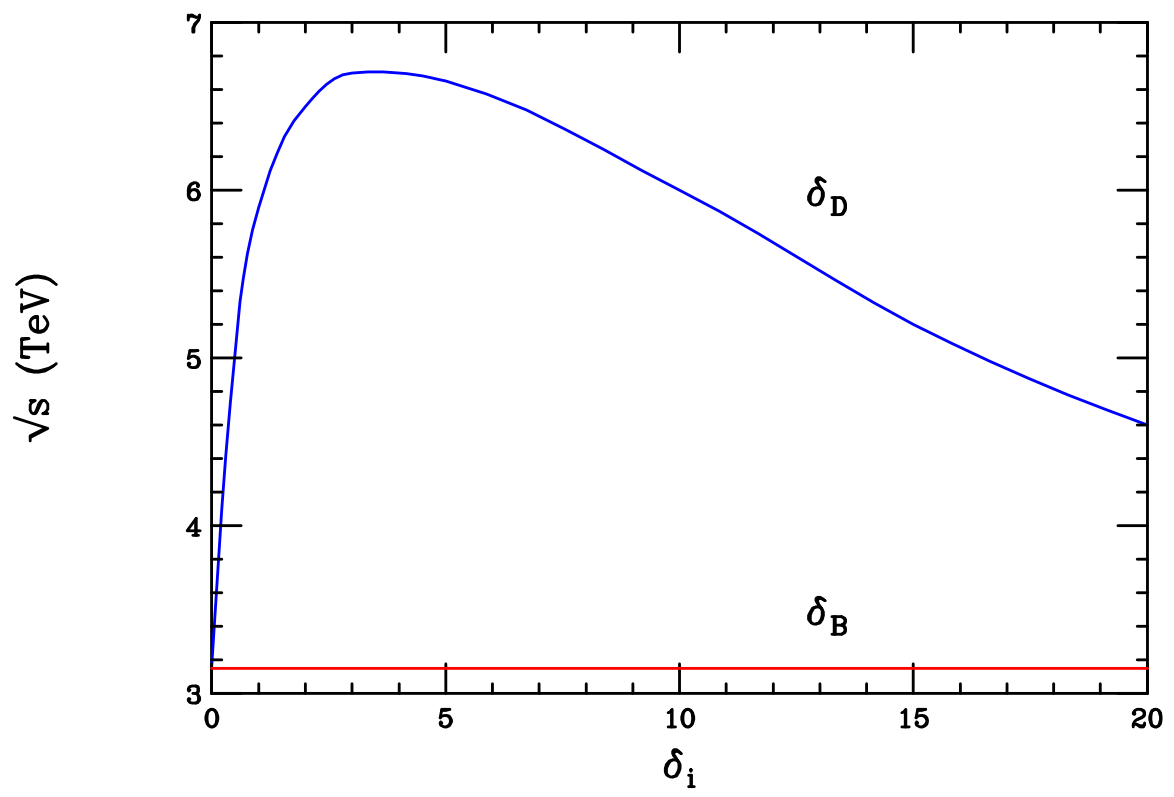

Figure 8: The scattering energy at which perturbative unitarity is violated in $W_{L}^{+} W_{L}^{-}$scattering as a function of the kinetic terms. We take $\kappa=1$.

A note about numerical instabilities is in order. We find that the sum rule governing the coefficient of the $s^{2}$ term is satisfied at the level of $10^{-6}$ after the first KK state is included, while the sum rule for the $s$ term is satisfied to the level of $10^{-2}$. After a few more 
states are included, the first sum rule is satisfied to $\mathcal{O}\left(10^{-9}\right)$, and the second to $\mathcal{O}\left(10^{-3}\right)$. This demonstrates that the PUV is due to incomplete cancellations in the term growing like $s$, as well as the presence of the constant term. However, consider the case where there is a numerical instability in the calculation of the couplings at the $10^{-8}$ level. Then we can estimate the energy scale at which this becomes important by noting that the amplitude goes like $10^{-8}\left(s^{2} / M_{W}^{4}\right)$. This becomes of order unity when $\sqrt{s} \sim 8 \mathrm{TeV}$, implying that a calculation good to only 8 digits will give incorrect results when the scale of PUV is in the few TeV range. Since unitarity depends on delicate cancellations, it could be expected that any error will decrease the scale of PUV. However, we have, somewhat surprisingly, found that this is not necessarily true. The reason is that the terms growing like $s$ and $s^{2}$ have the opposite sign. A numerical error can thus cause the $s^{2}$ term to turn on prematurely and cancel the contribution from the $s$ term, leading to an apparent scale of PUV higher than it actually is. For example, we studied one case where a numerical error at the level of $10^{-8}$ caused the apparent scale of PUV to be $12 \mathrm{TeV}$, while the correct scale was actually $6 \mathrm{TeV}$. For this reason, all our results were computed independently on two platforms, with agreement to greater than 12 digits.

\section{Conclusions}

The Warped Higgsless Model, which breaks the electroweak symmetry via boundary conditions associated with an extra dimension, offers a promising alternative to the Higgs mechanism. A custodial $S U(2)_{D}$ symmetry is present in the model, so that reasonable agreement with precision electroweak data is conceivable. However, the degree of such agreement varies as the parameter space of the model is explored, and some regions can be excluded. Here, we examined the effects of including $\operatorname{IR}(\mathrm{TeV})$-brane kinetic brane terms associated with the $U(1)_{B-L}$ and $S U(2)_{D}$ gauge symmetries of the model. We found that the addition of 
the $U(1)_{B-L}$ kinetic term enhances the agreement with the tree-level SM electroweak relations, particularly for larger values of the ratio of the $5-d$ couplings $g_{5 R} / g_{5 L}$, with reasonable values of the brane term parameter $\delta_{B}$. However, including the $S U(2)_{D}$ brane term alone results in a stark disagreement with the SM tree level relations in the electroweak sector. We performed a limited exploration of the full parameter space and found it is possible that a combination of the two IR-brane terms may result in a reasonable consistency with the tree-level SM relations.

In its original form, the WHM has some difficulties in the gauge sector in that perturbative unitarity is violated at the $\mathrm{TeV}$-scale in $W_{L}^{+} W_{L}^{-}$scattering. This does not exclude the model from being viable, but does suggest that interactions in the gauge sector are problematic. To restore unitarity in the gauge sector, additional new physics must be introduced. Here, we again examined the effects of including the $\operatorname{IR}(\mathrm{TeV})$-brane kinetic brane terms. It is well-known that the addition of brane terms can alter the couplings and masses of gauge KK states, and this would thus affect the KK contributions to $W_{L}^{+} W_{L}^{-}$scattering. While we found that the $U(1)_{B-L}$ brane term does modify the gauge KK spectrum, we also discovered that PUV in $W_{L}^{+} W_{L}^{-}$scattering is independent of such a brane term and hence remains unaffected by its presence. This is because this scattering process is inherently non-Abelian and should not depend on the $U(1)_{B-L}$ dynamics. In contrast, the inclusion of the $S U(2)_{D}$ kinetic term does affect $W_{L}^{+} W_{L}^{-}$scattering; for moderate values of the brane term, violation of perturbative unitarity is delayed until $\sqrt{s} \approx 7 \mathrm{TeV}$. In addition, we also investigated the collider bounds on the production of the lightest gauge KK excitation as a function of the brane terms. Searches for new gauge bosons at the Tevatron and LEP II exclude large values of the kinetic term parameters $\delta_{B, D}$.

Our analysis shows how various directions in the parameter space of the WHM affect its phenomenology. Requiring a model that is perturbatively sensible up to $\mathcal{O}(10) \mathrm{TeV}$ 
favors $\kappa=1$ and $1 \lesssim \delta_{D} \lesssim 10$, regardless of the size of $\delta_{B}$. Collider constraints on the KK modes of the gauge bosons can accommodate this range of parameters, as long as $\delta_{B, D} \lesssim 2-3$, with the Tevatron bounds depending on the fermion localization. We observe that the requirements of multi- $\mathrm{TeV}$ perturbative unitarity and those imposed by tree level SM relations, as represented by the pseudo-oblique parameters and various values of $\sin ^{2} \theta$, do not coexist without tension in this model. However, a direct comparison of these latter quantities with the electroweak data requires a computation of the radiative corrections in the WHM, which lies outside the scope of this work. Thus, it remains an open possibility that this model could provide a viable alternative for electroweak symmetry breaking, valid far above the weak scale.

\section{Acknowledgements}

We would like to thank Csabi Csaki, Graham Kribs, Hitoshi Murayama, and John Terning for discussions related to this work. The work of H.D. was supported by the Department of Energy under grant DE-FG02-90ER40542.

\section{References}

[1] C. Csaki, C. Grojean, H. Murayama, L. Pilo and J. Terning, "Gauge theories on an interval: Unitarity without a Higgs," arXiv:hep-ph/0305237.

[2] C. Csaki, C. Grojean, L. Pilo and J. Terning, "Towards a realistic model of Higgsless electroweak symmetry breaking," arXiv:hep-ph/0308038.

[3] L. Randall and R. Sundrum, Phys. Rev. Lett. 83, 3370 (1999) arXiv:hep-ph/9905221.

[4] J. M. Maldacena, Adv. Theor. Math. Phys. 2, 231 (1998) [Int. J. Theor. Phys. 38, 1113 (1999)] arXiv:hep-th/9711200. 
[5] Y. Nomura, "Higgsless theory of electroweak symmetry breaking from warped space," arXiv:hep-ph/0309189.

[6] R. Barbieri, A. Pomarol and R. Rattazzi, "Weakly coupled Higgsless theories and precision electroweak tests," arXiv:hep-ph/0310285.

[7] G. Burdman and Y. Nomura, "Holographic theories of electroweak symmetry breaking without a Higgs boson," arXiv:hep-ph/0312247.

[8] K. Agashe, A. Delgado, M. J. May and R. Sundrum, JHEP 0308, 050 (2003) arXiv:hep-ph/0308036.

[9] H. Davoudiasl, J. L. Hewett, B. Lillie and T. G. Rizzo, "Higgsless electroweak symmetry breaking in warped backgrounds: Constraints and signatures," arXiv:hep-ph/0312193.

[10] G. Cacciapaglia, C. Csaki, C. Grojean and J. Terning, "Oblique corrections from Higgsless models in warped space," arXiv:hep-ph/0401160.

[11] M. Carena, T. M. P. Tait and C. E. M. Wagner, Acta Phys. Polon. B 33, 2355 (2002) arXiv:hep-ph/0207056 ; H. Davoudiasl, J. L. Hewett and T. G. Rizzo, Phys. Rev. D 68, 045002 (2003) arXiv:hep-ph/0212279; JHEP 0308, 034 (2003) arXiv:hep-ph/0305086; M. Carena, E. Ponton, T. M. P. Tait and C. E. M. Wagner, Phys. Rev. D 67, 096006 (2003) arXiv:hep-ph/0212307.

[12] T.Affolder et al., CDF Collaboration, Phys. Rev. Lett. 87, 231803 (2001).

[13] See, for example, the talk given by T. Nelson, CDF Collaboration, SLAC, Jan. 2004.

[14] The analysis of LEPII data leading to bounds on new gauge boson-like signatures can be found in C. Geweniger et al., LEP Electroweak Working Group note LEP2FF/02$03(2002)$ 
[15] M. J. Duncan, G. L. Kane and W. W. Repko, Nucl. Phys. B 272, 517 (1986). 